\title{
Intercellular adhesion molecule- 1 and $S-100 \beta$ in sevoflurane combined with epidural anesthesia for radical resection of lung cancer
}

\author{
NAN ZHAO ${ }^{1}$, CHUNYUAN LU $^{2}$, LIANG LIU $^{1}$, PENG SUN $^{1}$ and JIANGE HAN ${ }^{1}$ \\ ${ }^{1}$ Department of Anesthesiology, Tianjin Chest Hospital, Tianjin 300222; ${ }^{2}$ Department of Anesthesiology, \\ The Second Hospital of Tianjin Medical University, Tianjin 300211, P.R. China
}

Received November 1, 2019; Accepted December 13, 2019

DOI: $10.3892 / \mathrm{ol} .2019 .11245$

\begin{abstract}
Significance of intercellular adhesion molecule-1 (ICAM-1) and S-100 $\beta$ protein (S-100 $\beta$ ) were investigated in sevoflurane combined with epidural anesthesia for radical resection of lung cancer. In total, 172 patients who underwent radical resection of lung cancer from May 2014 to January 2016 and 160 blood samples from healthy patients in the same period were selected for prospective analysis. Lung cancer patients were the therapy group (TG). Venous blood $(2 \mathrm{ml})$ was taken from patients before anesthesia (T1) and after anesthesia at $30 \mathrm{~min}$ (T2), $3 \mathrm{~h}$ (T3) and $24 \mathrm{~h}$ (T4), respectively. Healthy physical examination samples were the control group (CG). Enzyme linked immunosorbent assay (ELISA) was used to detect the concentration of ICAM-1 and RT-PCR to detect the concentration of S-100 $\beta$. The changes of ICAM-1 and S-100 $\beta$ concentrations and their significance to patients were analyzed. The serum ICAM-1 and S-100 $\beta$ concentrations in TG were significantly higher than those in $\mathrm{CG}(\mathrm{P}<0.001)$, and were the highest at $\mathrm{T} 1(\mathrm{P}>0.001)$, followed by T2 $(\mathrm{P}<0.001)$. Of the 172 patients 27 cases had adverse complications during the perioperative period. Patients were divided into the ICAM-1 high concentration group (CHCG), the ICAM-1 low concentration group (CLCG) and the S-100 $\beta$ high concentration group (SHCG) and the S-100 $\beta$ low concentration group (SLCG). The 3-year survival rate of CHCG was significantly lower than CLCG and SLCG $(\mathrm{P}<0.001)$. ICAM-1 and S-100 $\beta$ protein in sevoflurane combined with epidural anesthesia for radical resection of lung cancer can effectively predict the perioperative adverse complications of patients, and have better monitoring significance for the prognosis of patients.
\end{abstract}

Correspondence to: Dr Jiange Han, Department of Anesthesiology, Tianjin Chest Hospital, 261 Taierzhuang South Road, Tianjin 300222, P.R. China

E-mail: gcc9n2@163.com

Key words: ICAM-1, S-100 $\beta$, radical resection of lung cancer, sevoflurane combined with epidural anesthesia

\section{Introduction}

Lung cancer is the main cause of cancer death. The incidence rate and mortality rate are the largest proportion of malignant tumors in China (1). According to statistics, there were 1.82 million new lung cancer cases and 1.59 million deaths worldwide in 2012 (2). The global prevalence of tobacco has led to a rapid increase in new lung cancer cases and deaths. It is estimated that the death toll from lung cancer in the world will increase to 3 million by 2035, with men increasing from 1.1 million in 2012 to 2.1 million in 2035 and women from 500,000 in 2012 to 900,000 in 2035 (3). Second-hand tobacco smoke is also an important cause of lung cancer. It is estimated that 21,400 non-smokers die of lung cancer every year (4). The extremely high morbidity and mortality rate of lung cancer has caused heavy economic and health burden. Although diagnosis and treatment have made great progress, the 5-year overall survival rate is only approximately $15 \%$ (5), with poor prognosis. Therefore, the diagnosis, treatment and prognosis of lung cancer are still highly concerning issues.

At present, chemotherapy, radiotherapy and radical resection of lung cancer are the main treatments for lung cancer in clinic (6). Radical resection of lung cancer is the first choice for patients who can be treated surgically and can accept surgical risks although the risk is relatively high (7). Anesthesia is the decisive factor for the success of the operation, sevoflurane inhalation anesthesia is the commonly used anesthesia method for thoracotomy, and combined epidural anesthesia has higher hemodynamic stability (8). Cognitive dysfunction is a common adverse complication after anesthesia surgery. Studies have found that $S-100 \beta$ is a calcium binding protein of low molecular weight and is found in glial cells of central and peripheral nervous systems $(9,10)$. $S-100 \beta$ in serum is at physiologically low concentration in healthy population, and not easy to detect, but the expression in serum of brain injured people is abnormally increased (11-13). The level of S-100 $\beta$ in patients' serum can evaluate cognitive function, but its prognostic value in lung cancer has not been studied. ICAM-1 is an adhesion molecule and a member of the immunoglobulin superfamily genes. It is related to cell migration and invasion, plays a role in tumor metastasis (14), and can be used as a prognostic factor for patients (15). 
This study examined the concentration changes of ICAM-1 and $\mathrm{S}-100 \beta$ in sevoflurane combined with epidural anesthesia for radical resection of lung cancer at different times to study the predictive value of the two proteins for perioperative adverse complications and patient prognosis.

\section{Patients and methods}

Collection of clinical data. In total, 172 lung cancer patients diagnosed and treated in Tianjin Chest Hospital (Tianjin, China) from May 2014 to January 2016 were collected as the therapy group (TG) and 160 normal healthy people who underwent physical examination in the hospital during the same period were collected as the control group (CG). The laboratory and imaging examinations of the patients in the CG were normal without allergic diseases and autoimmune diseases. This study was approved by the Ethics Committee of Tianjin Chest Hospital.

Inclusion and exclusion criteria of the patients. Inclusion criteria: The patients were diagnosed as non-small cell lung cancer by thoracoscopic pleural biopsy. The diagnostic criteria referred to the lung cancer guidelines of the American College of Chest Physicians in 2013 (10). Tumor diameter was $<5 \mathrm{~cm}$ and ASA-PS grade (16) was $\geq 3$. The patients did not undergo radiotherapy or chemotherapy and were able to tolerate onelung ventilation. The patients had complete clinical data. Patients and their families were informed and signed an informed consent form.

Exclusion criteria: hypovolemia, shock, infection at puncture site, mental disease, low coagulation, hepatic and kidney function obstacle. Patients and their families had concerns.

Anesthesia methods. Before the operation, $8 \mathrm{ml} / \mathrm{kg} / \mathrm{h}$ of lactated ringer's solution (item number: H10983055, Shanghai Baite Medical Product Co., Ltd.) was injected through the forearm vein approach. Electrocardiogram, heart rate $(\mathrm{HR})$, blood oxygen saturation $\left(\mathrm{SpO}_{2}\right)$ and double spectrum index (BIS) were monitored. Arterial pressure was monitored by right invasive arterial puncture and catheter check. Central venous pressure (CVP) was monitored by right internal jugular vein puncture and catheter check. Epidural puncture and catheterization were performed on $\mathrm{T} 7-8$, followed by $3 \mathrm{ml}$ and $2 \%$ lidocaine (Southwest Pharmaceutical Co., Ltd., SFDA approval no. H50020038). It was confirmed that there were no adverse reactions after anesthesia. Before skin incision for $30 \mathrm{~min}, 10 \mathrm{ml}$ of $0.25 \%$ ropivacaine (Guangdong Jiabo Pharmaceutical Co., Ltd., SFDA approval no. H20133178) and $2 \mu \mathrm{g} / \mathrm{ml}$ of fentanyl (Yichang Humanwell Pharmaceutical Co., Ltd., SFDA approval no. H20003688) were injected into the epidural space. Induction of general anesthesia was inhalation of $8 \%$ sevoflurane (Shanghai Hengrui Pharmaceutical Co., Ltd, SFDA approval no. H20070172) at a high flow rate (oxygen flow rate $8-101 / \mathrm{min}$ ). After losing consciousness, the oxygen flow rate was adjusted to $21 / \mathrm{min}$. The end tidal sevoflurane concentration was kept at $2 \%$. The tidal volume of mechanical ventilation was $6-8 \mathrm{ml} / \mathrm{kg}$. The respiratory frequency was 12 times $/ \mathrm{min}$, with the exhalation: inhalation $=1: 2$. The oxygen flow rate was $2.0 \mathrm{l} / \mathrm{min}$. Anesthesia maintenance was continuous inhalation of sevoflurane with an end tidal concentration of $1.5-3.5 \%$. Cisatracurium $(0.05-0.1 \mathrm{Mg} / \mathrm{kg}$ ) was intermittently intravenously injected (Jiangsu Hengrui Pharmaceutical Co., Ltd., SFDA approval no. H20060869). End tidal $\mathrm{CO}_{2}$ partial pressure was $30-40 \mathrm{mmHg}$, BIS was 40-60 and CVP was $\leq 12 \mathrm{~cm} \mathrm{H}_{2} \mathrm{O}$. The fluctuation of blood pressure and HR did not exceed $20 \%$ of the baseline. Before the end of the operation for $30 \mathrm{~min}$, the muscle relaxant was stopped and $10 \mathrm{ml}$ of mixed anesthetic solution was injected into the epidural space. Sevoflurane inhalation was stopped $10 \mathrm{~min}$ before the end of the operation. The airway was flushed with oxygen at a high flow rate $(4 \mathrm{ml} / \mathrm{min})$. PCIA was performed and VAS score $\leq 3$ was maintained. When swallowing reflux recovered, spontaneous respiration rate was $\geq 12$ times $/ \mathrm{min}, \mathrm{CO}_{2}$ partial pressure was $<45 \mathrm{mmHg}$ and $\mathrm{SpO}_{2}$ was $\geq 95 \%$. Tracheal catheter was pulled out. The patient was observed for $30 \mathrm{~min}$. If $\mathrm{SpO}_{2}$ was $<92 \%$, the oxygen mask was used at a speed of $5 \mathrm{l} / \mathrm{min}$. There were no routine preventive measures against nausea and vomiting. If symptoms worsen, drugs should be prescribed. The patient returned to the ward after reaching the corrected Aldrete score $\geq 9$ (17).

Sample collection and detection. Venous blood $(2 \mathrm{ml})$ was taken from the $\mathrm{CG}$ at fasting and the four time points of T1, T2, T3 and T4. The blood was allowed to stand at room temperature for $30 \mathrm{~min}$ and centrifuged at $3,000 \mathrm{x}$ at $4^{\circ} \mathrm{C}$ for $10 \mathrm{~min}$. The supernatant was frozen in a refrigerator at $-80^{\circ} \mathrm{C}$ for centralized detection.

Detection of ICAM-1 concentration in serum by ELISA. Blank, standard and sample wells to be tested were set up. The SO standard sample with a concentration of 0 was added into the blank well. The standard sample was added into the standard well. The sample well was first added with the sample to be tested. Sample diluent and horseradish peroxidase (HRP) labeled detection antibody were added to all micropores except the sample well. The unbound biotinylation antibodies were removed by full washing. HRP-labeled avidin was added and TMB substrates were added to color after washing again. TMB turned blue under catalysis and yellow under the action of acid. The absorbance (OD value) was measured by enzyme micro-plate reader at $450 \mathrm{~nm}$ wavelength. The corresponding concentration was converted from the standard curve.

Detection of $S-100 \beta$ concentration in serum by RT-PCR. The collected serum was extracted with TRIzol kit (Invitrogen ${ }^{\mathrm{TM}}$; Thermo Fisher Scientific, Inc., 15596026) for total RNA. The purity, concentration and integrity of the extracted total RNA were detected with ultraviolet spectrophotometer and agarose gel electrophoresis. 5X TransScript ${ }^{\circledR}$ II All-in-One SpuperMix for qPCR and gDNA Remover kit (belonging to TransScript Green two-step qrt-pcr SuperMix) were used for reverse transcription. The operation procedure was strictly in accordance with the manufacturer's kit. Then TransScript Green Two-Step qRT-PCR SuperMix (China TransGen Biotech, AQ201-01) and ABI7500 (Applied Biosystems ${ }^{\mathrm{TM}}$; Thermo Fisher Scientific, Inc.) were used for PCR amplification. PCR reaction system was $1 \mu \mathrm{l}$ of cDNA, each $0.4 \mu \mathrm{l}$ of upstream and downstream primers, $10 \mu \mathrm{l}$ of $2 \mathrm{X}$ TransScript ${ }^{\circledR}$ Tip Green qPCR SuperMix 
Table I. Baseline data.

\begin{tabular}{|c|c|c|c|c|}
\hline Factors & TG $(n=172)$ & $\mathrm{CG}(\mathrm{n}=160)$ & $\mathrm{t} / \chi^{2}$ value & P-value \\
\hline Age & $60.2 \pm 9.7$ & $58.9 \pm 11.2$ & 1.133 & 0.258 \\
\hline \multicolumn{5}{|l|}{ Sex } \\
\hline Male & $115(66.86)$ & $108(67.50)$ & 0.015 & 0.901 \\
\hline Female & $57(33.14)$ & $52(32.50)$ & & \\
\hline BMI $\left(\mathrm{kg} / \mathrm{m}^{2}\right)$ & $21.8 \pm 1.6$ & $22.1 \pm 1.2$ & 1.922 & 0.056 \\
\hline Place of residence & & & 0.106 & 0.745 \\
\hline City & $88(51.16)$ & $79(49.38)$ & & \\
\hline Rural & $84(48.84)$ & $81(50.62)$ & & \\
\hline \multicolumn{5}{|l|}{ Differentiation degree } \\
\hline Poorly differentiated & $54(31.40)$ & $0(0.00)$ & & \\
\hline Medium + well differentiated & $118(68.60)$ & $0(0.00)$ & & \\
\hline \multicolumn{5}{|l|}{ Lymphatic metastasis } \\
\hline Metastasis & $124(72.09)$ & $0(0.00)$ & & \\
\hline Non-metastasis & $48(27.91)$ & $0(0.00)$ & & \\
\hline \multicolumn{5}{|l|}{ TNM stages } \\
\hline $\mathrm{T} 1$ & $67(38.40)$ & $0(0.00)$ & & \\
\hline $\mathrm{T} 2$ & $105(61.6)$ & $0(0.00)$ & & \\
\hline
\end{tabular}

TG, therapy group; CG, control group; BMI, body mass index; TNM, tumor-node-metastasis.

and Passive Reference Dye (50X). Finally, Nuclease-free Water was added to replenish to $20 \mu \mathrm{l}$. PCR reaction conditions were pre-degeneration at $94^{\circ} \mathrm{C}$ for $30 \mathrm{sec}$, degeneration at $94^{\circ} \mathrm{C}$ for $5 \mathrm{sec}$, annealing and extention at $60^{\circ} \mathrm{C}$ for $30 \mathrm{sec}$, then followed a total of 40 cycles. Then 3 repeated wells were set up of each sample. The experiment was carried out 3 times. In this study, GAPDH was used as internal parameters. $2^{-\Delta \Delta \mathrm{Ct}}$ was used to analyze the data.

Follow-up of patients. The survival of the patients was followed up for 3 years through outpatients' reexamination and by telephone. Follow-up time was 1, 3, 6, 12, 24, 36 months from the patients' radical resection of lung cancer to the follow-up time or the death of the patients.

Observation indexes. Main outcome measures: The concentration changes of ICAM-1 and S-100 $\beta$ before and after treatment. The predictive value of ICAM-1 and S-100 $\beta$ for adverse complications during perioperative period. The predictive value of ICAM-1 and S-100 $\beta$ for death of patient prognosis within 3 years.

Secondary observation index: The survival curve was drawn according to the survival of the patients. The median concentration of ICAM- 1 and S-100 $\beta$ before treatment was taken and divided into high and low expression groups to draw the survival curve of the patients.

Statistical analysis. In this study, SPSS20.0 (Shanghai Cabe Information Technology Co., Ltd.) was used to carry out statistical analysis on the collected data. GraphPad Prism 7 (Shenzhen Qiruitian Software Technology Co., Ltd.) was used to draw the illustrations. Counting data was expressed as a percentage (\%). Chi-square test was used, and expressed as $\chi^{2}$. All data conformed to normal distribution and were expressed by mean number \pm standard deviation (mean $\pm \mathrm{SD}$ ). The independent sample t-test was used for inter-group comparisons. Multiple time points were expressed by repetitive measurement and analysis of variance, expressed as $\mathrm{F}$. Then Bonferroni test was used for inspection. $\mathrm{P}<0.05$ was considered to indicate a statistically significant difference.

\section{Results}

Comparison of baseline data of patients in both groups. According to the data in Table I, there was no significant difference in age, sex, BMI and place of residence between the TG and CG $(\mathrm{P}>0.05)$, which were comparable.

Comparison of ICAM-1 and S-100 $\beta$ concentrations of patients in both groups. Comparing the concentration of ICAM-1 and $\mathrm{S}-100 \beta$ in the serum of the TG and the CG before treatment, it was found that the concentration of ICAM-1 in the serum of TG (283.174 \pm 31.543$)$ was significantly higher than that of CG $(119.281 \pm 24.615)(\mathrm{P}<0.001)$. The concentration of $\mathrm{S}-100 \beta$ in the serum of TG $(1.412 \pm 0.147)$ was significantly higher than that of CG $(1.031 \pm 0.112)(\mathrm{P}<0.001)$, as shown in Fig. 1. The concentration of ICAM-1 in TG was highest at T1 $(283.174 \pm 31.543)$ $(\mathrm{P}<0.001)$, followed by T2 $(247.246 \pm 28.714)(\mathrm{P}<0.001)$. The concentration of ICAM-1 at T3 $(225.436 \pm 27.827)$ was higher than T4 (174.113 \pm 29.281$)(\mathrm{P}<0.001)$. In $\mathrm{TG}$, the concentration of $\mathrm{S}-100 \beta$ at $\mathrm{T} 1(1.412 \pm 0.147)$ was the highest $(\mathrm{P}<0.001)$, followed by T2 $(1.358 \pm 0.154)(\mathrm{P}<0.001)$. The concentration of $\mathrm{S}-100 \beta$ at T3 $(1.236 \pm 0.161)$ was higher than T4 $(1.124 \pm 0.165)$ $(\mathrm{P}<0.001)$, as shown in Fig. 2. 

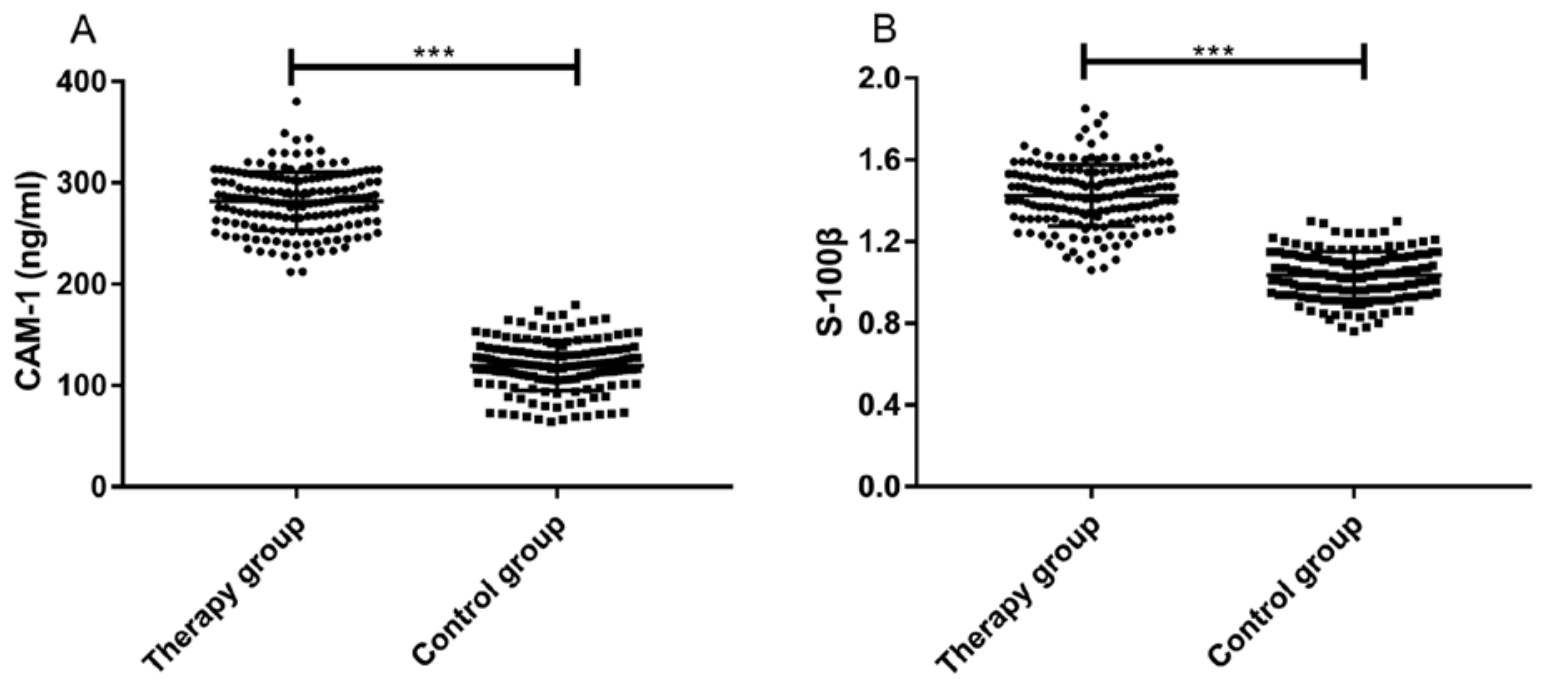

Figure 1. The expression levels of (A) ICAM-1 and (B) S-100 $\beta$ in serum before treatment in the two groups. Expression levels of ICAM-1 in serum before treatment in the two groups. Concentration of serum ICAM-1 in TG $(283.174 \pm 31.543)$ was significantly higher than that in CG $(119.281 \pm 24.615)(\mathrm{P}<0.001)$. The relative expression levels of S-100 $\beta$ in serum before treatment in the two groups. Concentration of serum S-100 $\beta$ in TG $(1.412 \pm 0.147)$ was significantly higher than that in CG $(1.031 \pm 0.112)(\mathrm{P}<0.001) .{ }^{* * *} \mathrm{P}<0.001$. ICAM-1, intercellular adhesion molecule-1; S-100 $\beta, \mathrm{S}-100 \beta$ protein; TG, therapy group; CG, control group.
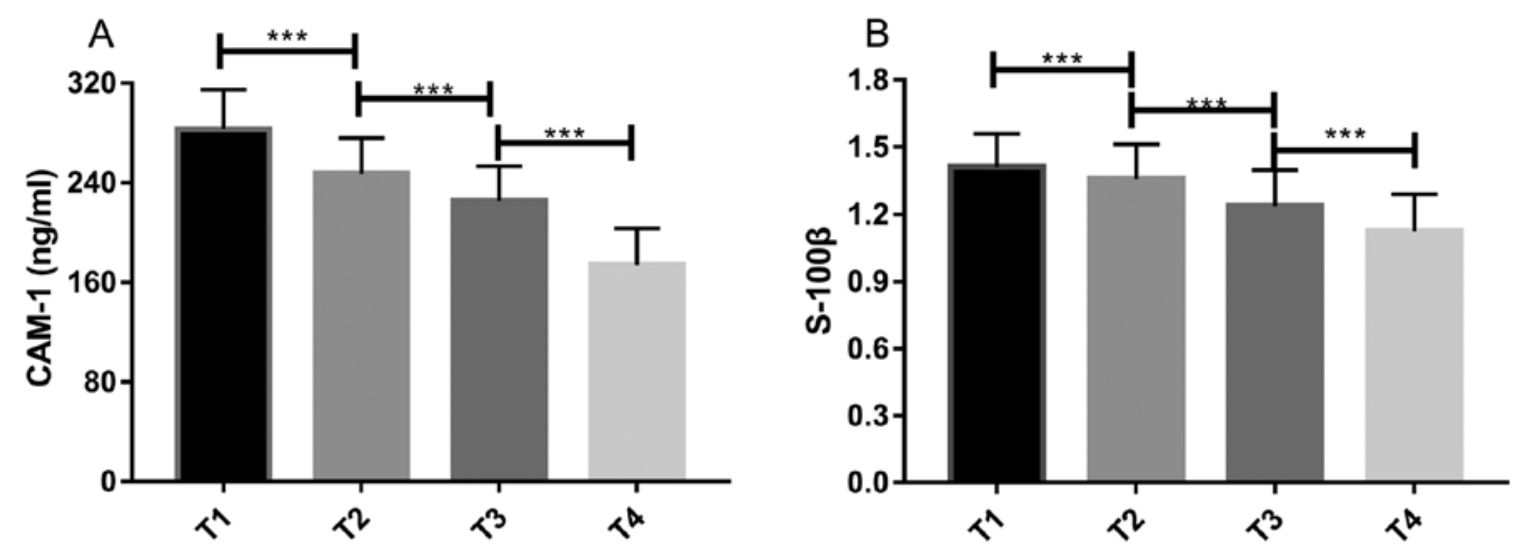

Figure 2. Changes in the expression levels of ICAM-1 and S-100 $\beta$ in patients after anesthesia for $24 \mathrm{~h}$. (A) Concentration of ICAM-1 was highest at T1 (283.174 \pm 31.543$)$, followed by T2 (247.246 \pm 28.714$)$. Concentration of ICAM-1 at T3 $(225.436 \pm 27.827)$ was higher than T4 (174.113 \pm 29.281$)$. (B) Concentration of S-100 $\beta$ at T1 $(1.412 \pm 0.147)$ was the highest $(\mathrm{P}<0.001)$, followed by T2 (1.358 \pm 0.154$)$. Concentration of S-100 $\beta$ at T3 $(1.236 \pm 0.161)$ was higher than T4 (1.124 \pm 0.165$) .{ }^{* * *} \mathrm{P}<0.001$. ICAM-1, intercellular adhesion molecule-1; S-100 $\beta, \mathrm{S}-100 \beta$ protein.

The predictive value of ICAM-1 and $S-100 \beta$ for perioperative adverse complications of patients. According to statistics, a total of 27 patients in TG had cognitive dysfunction during the perioperative period. Patients with adverse complications and patients without adverse complications were divided into two groups. Receiver operating characteristics (ROC) curves were drawn according to the concentrations of ICAM-1 and S-100 $\beta$ in the serum of patients in the two groups before treatment. The results showed that the area under ICAM-1 curve was 0.906 and the youden index was $81.38 \%$. When cut-off value was $>266$, the sensitivity and specificity of ICAM-1 in predicting perioperative adverse complications were 81.38 and $100 \%$, respectively. The area under the S-100 $\beta$ curve was 0.899 and the youden index was $76.55 \%$. When cut-off value was $>1.375$, the sensitivity and specificity of $\mathrm{S}-100 \beta$ in predicting perioperative adverse complications were 76.55 and $100 \%$, respectively, as shown in Fig. 3.
Relationship between survival of patients, ICAM-1 and $S-100 \beta$. According to statistics on the overall survival rate of the patients, the results showed that the 172 patients were followed up without any defects and the overall survival rate of the patients was $37.60 \%$. According to the median concentration of ICAM- 1 and S-100 $\beta$ before treatment, the patients were divided into high and low concentration groups, respectively. Observation on the overall survival rate of the two groups showed that the overall survival rate of the ICAM-1 low concentration group (CLCG) was significantly higher than that of the ICAM-1 high concentration group (CHCG). There was a significant difference in survival between the two groups $(\mathrm{P}=0.001)$. The overall survival rate of the $\mathrm{S}-100 \beta$ low concentration group (SLCG) was significantly higher than that of the S-100 $\beta$ high concentration group (SHCG). There was a significant difference in survival between the two groups $(\mathrm{P}=0.026)$. The deceased and the surviving patients after the follow-up were divided into two groups. ROC curves were 


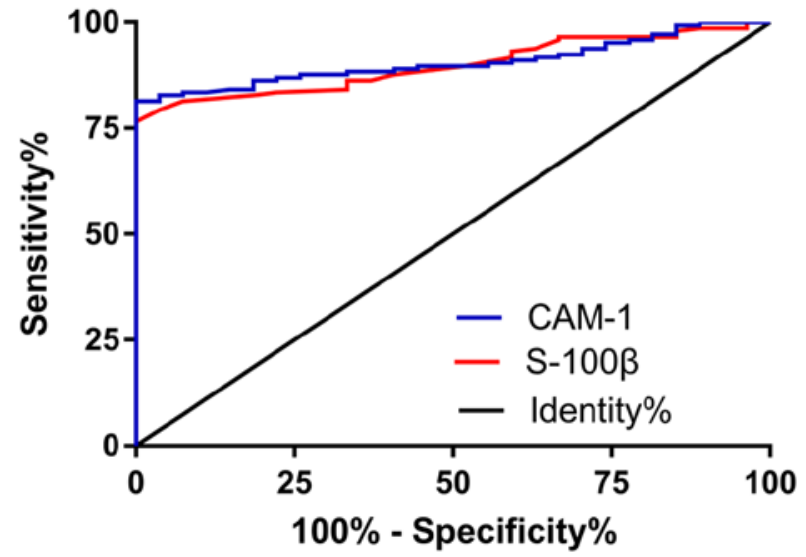

Figure 3. The predictive value of ICAM-1 and S-100 $\beta$ for perioperative adverse complications of patients. The red line is the ROC curve of S-100 $\beta$ for predicting perioperative adverse complications of patients. The area under the curve was 0.906 and the youden index was $81.38 \%$. When cutoff value was $>266$, the sensitivity and specificity of ICAM-1 in predicting perioperative adverse complications were 81.38 and $100 \%$, respectively. The blue line is the ROC curve of ICAM-1 for predicting perioperative adverse complications of patients. The area under the curve was 0.899 and the youden index was $76.55 \%$. When cut-off value was $>1.375$, the sensitivity and specificity of S-100 $\beta$ in predicting perioperative adverse complications were 76.55 and $100 \%$, respectively. ICAM-1, intercellular adhesion molecule $-1 ; \mathrm{S}-100 \beta$, S-100 $\beta$ protein; ROC, receiver operating characteristics.

drawn according to the concentrations of ICAM- 1 and S-100 $\beta$ in the serum of the two groups before treatment. The results showed that the area under ICAM- 1 curve was 0.864 and the youden index was $73.60 \%$. When cut-off value was $>280.9$, the sensitivity and specificity of ICAM-1 in predicting the prognosis of patients with death within 3 years were 73.60 and $100 \%$, respectively. The area under the S-100 $\beta$ curve was 0.804 and the youden index was $60 \%$. When cut-off value was $>1.465$, the sensitivity and specificity of S-100 $\beta$ in predicting the prognosis of patients with death within 3 years were 60 and 100\%, respectively, as shown in Figs. 4 and 5.

\section{Discussion}

With the prevalence of tobacco, lung cancer has changed from a rare disease to a global and a public health problem. From the perspective of public health, effective tobacco control is the main strategy to prevent lung cancer. Although smoking rate has begun to decline, lung cancer patients are still increasing year by year. According to a study from South Korean, the number of people who have never smoked with lung cancer has increased (18). Therefore, the treatment and prognosis of lung cancer is still a growing concern in the whole society.

At present, chemotherapy, radiotherapy and lobectomy are the main treatments for lung cancer in clinic. Surgery is the best way to treat lung cancer for patients who meet the surgical indications. Studies have found that at least $30 \%$ of patients with primary resectable non-small cell lung cancer will relapse within 5 years after treatment (19). Relapse is the main decisive factor of patient death. Therefore, it may improve treatment allocation to find a good prognosis index, thus providing adjuvant treatment for patients with high recurrence risk after surgery. Inflammation is an important component of tumor progression. Neutrophils and lymphocytes are commonly used
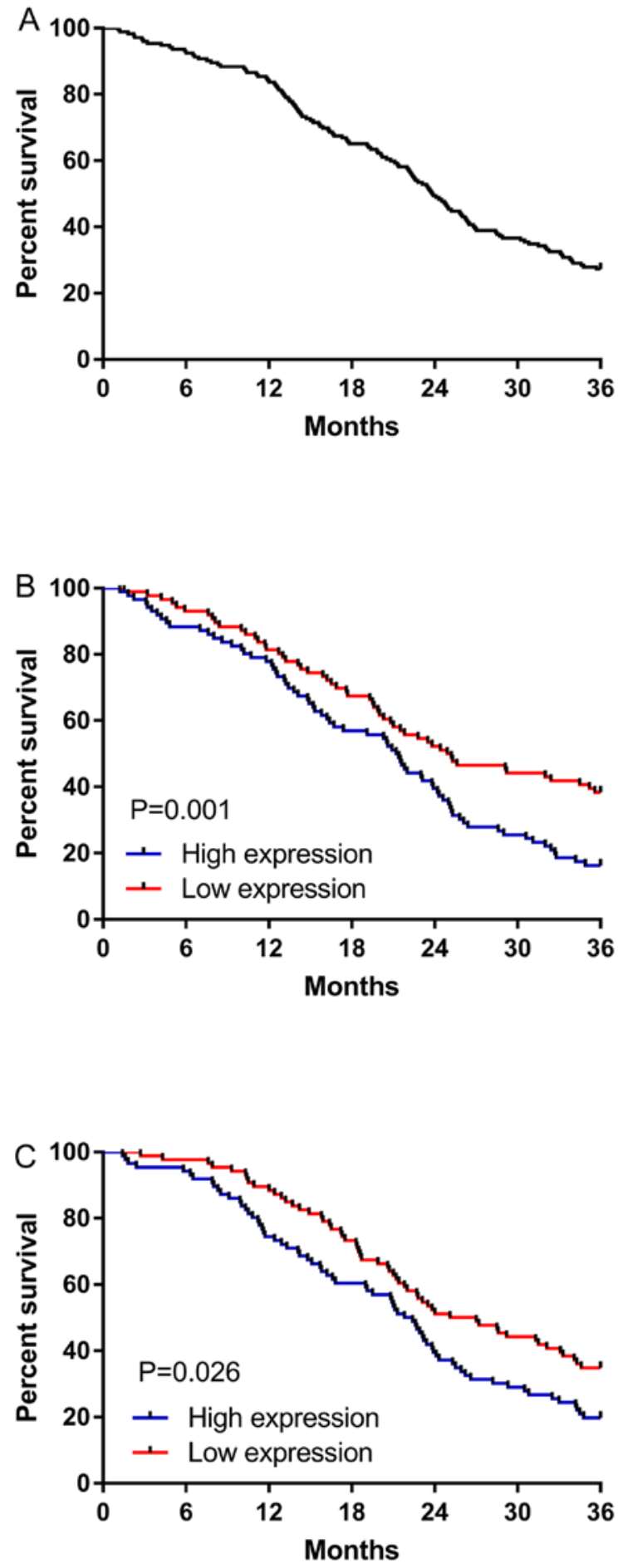

Figure 4. Patients' 3-year survival after treatment. (A) Patients' 3-year overall survival after treatment. (B) The overall survival rate of CLCG was significantly higher than that of $\mathrm{CHCG}$. There was a significant difference in survival between the two groups $(\mathrm{P}=0.001)$. (C) Overall survival rate of SLCG was significantly higher than that of SHCG. There was a significant difference in survival between the two groups $(\mathrm{P}=0.026)$. CLCG, ICAM-1 low concentration group; CHCG, ICAM-1 high concentration group; SHCG, S-100 $\beta$ high concentration group.

as prognostic indicators for lung cancer patients in clinic (20). However, tumor-related adverse reactions, such as anorexia, can also promote the expression of inflammatory factors, which cannot fully prove the biological basis of systemic inflammatory prognosis. Therefore, it is particularly important 


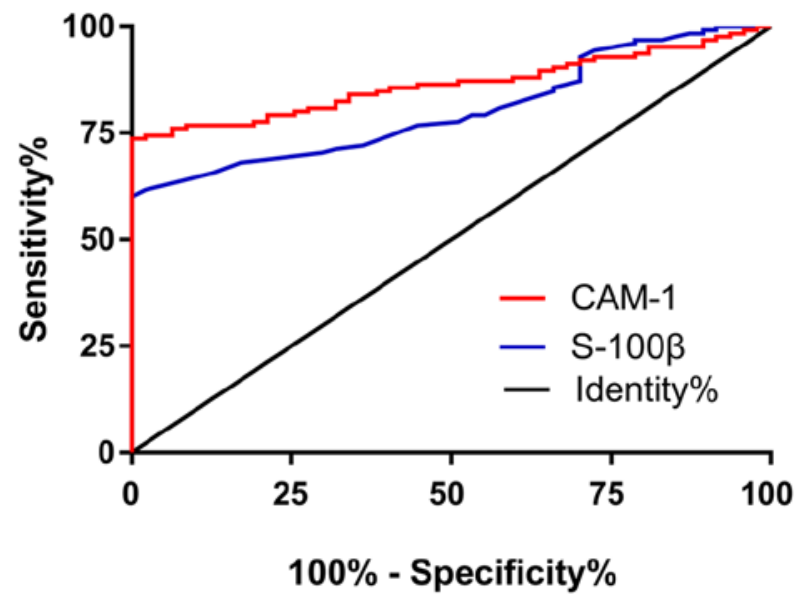

Figure 5. Predictive value of ICAM-1 and S-100 $\beta$ for patient survival after treatment. The red line is the ROC curve of ICAM-1 for predicting patient survival after treatment. The area under the curve was 0.864 and the youden index was $73.60 \%$. When cut-off value was $>280.9$, the sensitivity and specificity of ICAM-1 in predicting the prognosis of patients with death within 3 years were 73.60 and $100 \%$, respectively. The blue line is the ROC curve of $\mathrm{S}-100 \beta$ for predicting patient survival after treatment. The area under the curve was 0.804 and the youden index was $60.00 \%$. When cut-off value was $>1.465$, the sensitivity and specificity of S-100 $\beta$ in predicting the prognosis of patients with death within 3 years were 60 and $100 \%$, respectively. ICAM-1, intercellular adhesion molecule $-1 ; \mathrm{S}-100 \beta, \mathrm{S}-100 \beta$ protein; ROC, receiver operating characteristics.

to find new serological prognostic indicators to improve the current situation. Adhesion of tumor cells to endothelial cells by ICAM-1 is a key step in metastasis, which indicates poor prognosis of lung cancer patients to a large extent. However, cognitive dysfunction usually occurs after surgery anesthesia. Studies have found that $\mathrm{S}-100 \beta$ can be used as a prognostic indicator for patients with craniocerebral injury (21), but there are few studies on it as a prognostic indicator for lung cancer. Therefore, this study examined the concentration changes of ICAM-1 and S-100 $\beta$ in sevoflurane combined with epidural anesthesia for radical resection of lung cancer at different times to study the predictive value of the two proteins for perioperative adverse complications and patient prognosis.

In this study, we found that the concentration of ICAM-1 and $\mathrm{S}-100 \beta$ in patients' serum was significantly higher than that in CG by comparing the concentration of ICAM-1 and $\mathrm{S}-100 \beta$ in the serum of the two groups before treatment. The differential expression of the two proteins in patients and healthy people suggested that they may be used as diagnostic indicators for lung cancer. However, because this study focused on its significance for the prognosis of patients, the diagnostic value has not been thoroughly studied. Then we detected the concentration of ICAM- 1 and S-100 $\beta$ in patients' serum at T1, T2, T3 and T4. It was found that the concentration of ICAM-1 and S-100 $\beta$ decreased gradually. Finzel et al (22) found that after blocking endothelial soluble ICAM-1 with monoclonal antibody, its expression levels and adhesion decreased significantly. It was basically consistent with our research results. The results of Qiao et al (23) showed that the concentration of S-100 $\beta$ increased after sevoflurane anesthesia alone, while this research showed that the concentration gradually decreased. We speculated that the reason was that the operation combined with epidural puncture anesthesia reduced the stress response of patients and the cognitive dysfunction.

Anesthetic surgery is usually accompanied by adverse complications. This study counted 27 cases of adverse complications during perioperative period. Patients were divided into two groups according to whether adverse complications occurred or not. ROC curves were drawn according to the concentrations of ICAM- 1 and S-100 $\beta$ in the serum of patients in the two groups. The results showed that ICAM-1 and S-100 $\beta$ had the best prediction specificity and sensitivity when cut-off values were $>266$ and $>1.375$, respectively, which suggested that ICAM-1 and S-100 $\beta$ might be used as indicators to predict the occurrence of adverse complications in patients during perioperative period. The high expression of ICAM-1 and S-100 $\beta$ indicates that tumor metastasis is accelerated, brain injury is serious and it is significantly reduced after treatment. According to the results of this study, it also showed that it has high value in predicting adverse complications during the perioperative period.

The prognosis of lung cancer patients is poor and the 5 -year survival rate is less than $18 \%$ (24). According to our statistics, the 3 -year overall survival rate was only $37.6 \%$. Although the treatment of lung cancer has made great progress, the treatment results are still unsatisfactory due to late diagnosis, older patients, concomitant diseases and limited treatment options (25). According to the median concentration of ICAM-1 and S-100 $\beta$ before treatment, the patients were divided into high and low concentration groups. The value of the two indexes in patients' survival was observed. The results showed that the overall survival rate of CLCG and SLCG was significantly higher than that of CHCG and SHCG. There was a significant difference in survival between the two groups. It indicated that the two indexes had predictive value in patients' short-term prognosis and could be used as potential survival predictive indexes of patients. Therefore, we further drew ROC curves for the survival of patients. The results showed that when the concentrations of ICAM-1 and S-100 $\beta$ were $>280.9$ and $>1.465$ respectively, the prediction sensitivity was $73.60 \%$ and $60 \%$, respectively, and the specificity was $100 \%$. We speculate that the reason may be that the concentration of ICAM-1 and S-100 $\beta$ decreased significantly after treatment, the adhesion decreased and the brain injury decreased, thus slowing down the metastasis of tumor cells and improving the disease. Moreover, patients with low concentration of ICAM-1 and S-100 $\beta$ before treatment have better efficacy than patients with high concentration. Therefore, we speculate that high or low concentration of ICAM-1 and S-100 $\beta$ before treatment can predict the survival of patients. The value of ICAM-1 as a prognostic indicator was also reported by Kotteas et al (7).

Collectively, ICAM-1 and S-100 $\beta$ protein in sevoflurane combined with epidural anesthesia for radical resection of lung cancer can effectively predict the perioperative adverse complications of patients, and have better monitoring significance for the prognosis of patients.

\section{Acknowledgements}

Not applicable. 


\section{Funding}

No funding was received.

\section{Availability of data and materials}

The datasets used and/or analyzed during the current study are available from the corresponding author on reasonable request.

\section{Authors' contributions}

NZ wrote the manuscript and was responsible for anesthesia. CL conceived and designed the study. LL was responsible for the collection and analysis of the experimental data. PS interpreted the data and drafted the manuscript. JH detected S-100 $\beta$ concentration and revised the manuscript critically for important intellectual content. All authors read and approved the final manuscript.

\section{Ethics approval and consent to participate}

The study was approved by the Ethics Committee of Tianjin Chest Hospital (Tianjin, China). Patients who participated in this research had complete clinical data. Signed informed consents were obtained from the patients and/or the guardians.

\section{Patient consent for publication}

Not applicable.

\section{Competing interests}

The authors declare that they have no competing interests.

\section{References}

1. Chen W, Zheng R, Baade PD, Zhang S, Zeng H, Bray F, Jemal A, Yu XQ and He J: Cancer statistics in China, 2015. CA Cancer J Clin 66: 115-132, 2016.

2. Cheng TY, Cramb SM, Baade PD, Youlden DR, Nwogu C and Reid ME: The international epidemiology of lung cancer: Latest trends, disparities, and tumor characteristics. J Thorac Oncol 11: 1653-1671, 2016.

3. Didkowska J, Wojciechowska U, Mańczuk M and Łobaszewski J: Lung cancer epidemiology: Contemporary and future challenges worldwide. Ann Transl Med 4: 150, 2016.

4. Oberg M, Jaakkola MS, Woodward A, Peruga A and Prüss-Ustün A: Worldwide burden of disease from exposure to second-hand smoke: A retrospective analysis of data from 192 countries. Lancet 377: 139-146, 2011.

5. Molina JR, Yang P, Cassivi SD, Schild SE and Adjei AA: Non-small cell lung cancer: Epidemiology, risk factors, treatment, and survivorship. Mayo Clin Proc 83: 584-594, 2008.

6. Vansteenkiste J, De Ruysscher D, Eberhardt WE, Lim E, Senan S, Felip E and Peters S; ESMO Guidelines Working Group: Early and locally advanced non-small-cell lung cancer (NSCLC): ESMO Clinical Practice Guidelines for diagnosis, treatment and follow-up. Ann Oncol 24 (Suppl 6): vi89-vi98, 2013.

7. Kotteas EA, Gkiozos I, Tsagkouli S, Bastas A, Ntanos I, Saif MW and Syrigos KN: Soluble ICAM-1 levels in small-cell lung cancer: Prognostic value for survival and predictive significance for response during chemotherapy. Med Oncol 30: 662, 2013.

8. Zhao L, Liu L, Dong Z and Xiong J: miR-149 suppresses human non-small cell lung cancer growth and metastasis by inhibiting the FOXM1/cyclin D1/MMP2 axis. Oncol Rep 38: 3522-3530, 2017.
9. Neselius S, Brisby H, Theodorsson A, Blennow K, Zetterberg H and Marcusson J: CSF-biomarkers in Olympic boxing: Diagnosis and effects of repetitive head trauma. PLoS One 7: e33606, 2012.

10. Rivera MP, Mehta AC and Wahidi MM: Establishing the diagnosis of lung cancer: Diagnosis and management of lung cancer, 3rd ed: American College of Chest Physicians evidence-based clinical practice guidelines. Chest 143 (Suppl): e142S-e165S, 2013.

11. Xianbao L, Hong Z, Xu Z, Chunfang Z and Dunjin C: Dexmedetomidine reduced cytokine release during postpartum bleeding-induced multiple organ dysfunction syndrome in rats. Mediators Inflamm 2013: 627831, 2013.

12. Qin B, Panickar KS and Anderson RA: Cinnamon polyphenols attenuate the hydrogen peroxide-induced down regulation of S100 $\beta$ secretion by regulating sirtuin 1 in C6 rat glioma cells. Life Sci 102: 72-79, 2014.

13. Ehrenreich H, Kästner A, Weissenborn K, Streeter J, Sperling S, Wang KK, Worthmann H, Hayes RL, von Ahsen N, Kastrup A, et al: Circulating damage marker profiles support a neuroprotective effect of erythropoietin in ischemic stroke patients. Mol Med 17: 1306-1310, 2011.

14. Zhao LH, Shi ZH, Yin NN and Zhou JX: Use of dexmedetomidine for prophylactic analgesia and sedation in delayed extubation patients after craniotomy: A study protocol and statistical analysis plan for a randomized controlled trial. Trials 14: 251, 2013

15. Marenholz I, Heizmann CW and Fritz G: S100 proteins in mouse and man: From evolution to function and pathology (including an update of the nomenclature). Biochem Biophys Res Commun 322: 1111-1122, 2004.

16. Sankar A, Johnson SR, Beattie WS, Tait G and Wijeysundera DN: Reliability of the American Society of Anesthesiologists physical status scale in clinical practice. Br J Anaesth 113: 424-432, 2014

17. Li R, Xu X, Wu XM, Xiong LZ, Guo QL, Lian QQ, Heng XH, Zhang Y and Qing EM: Effect of dexmedetomidine on responses to endotracheal extubation during recovery from general anesthesia in patients with hypertension: A multicenter, randomized, blind, placebo-controlled clinical trial. Chin J Anesthesiol 33: 397-401, 2013 (In Chinese).

18. Park JY and Jang SH: Epidemiology of lung cancer in Korea: Recent trends. Tuberc Respir Dis (Seoul) 79: 58-69, 2016.

19. Cedrés S, Torrejon D, Martínez A, Martinez P, Navarro A, Zamora E, Mulet-Margalef $\mathrm{N}$ and Felip E: Neutrophil to lymphocyte ratio (NLR) as an indicator of poor prognosis in stage IV non-small cell lung cancer. Clin Transl Oncol 14: 864-869, 2012.

20. Mercier E, Boutin A, Lauzier F, Fergusson DA, Simard JF, Zarychanski R, Moore L, McIntyre LA, Archambault P, Lamontagne F, et al: Predictive value of S-100 $\beta$ protein for prognosis in patients with moderate and severe traumatic brain injury: Systematic review and meta-analysis. BMJ 346 (apr04 1): f1757, 2013.

21. Pinato DJ, Shiner RJ, Seckl MJ, Stebbing J, Sharma R and Mauri FA: Prognostic performance of inflammation-based prognostic indices in primary operable non-small cell lung cancer. $\mathrm{Br}$ J Cancer 110: 1930-1935, 2014.

22. Finzel AH, Reininger AJ, Bode PA and Wurzinger LJ: ICAM-1 supports adhesion of human small-cell lung carcinoma to endothelial cells. Clin Exp Metastasis 21: 185-189, 2004.

23. Qiao Y, Feng H, Zhao T, Yan $\mathrm{H}$, Zhang $\mathrm{H}$ and Zhao $\mathrm{X}$ : Postoperative cognitive dysfunction after inhalational anesthesia in elderly patients undergoing major surgery: The influence of anesthetic technique, cerebral injury and systemic inflammation. BMC Anesthesiol 15: 154, 2015.

24. Zappa $C$ and Mousa SA: Non-small cell lung cancer: Current treatment and future advances. Transl Lung Cancer Res 5: 288-300, 2016.

25. Leppert W: Quality of life in patients with advanced lung cancer at home palliative care at the in-patient palliative care unit. Medycyna Paliatywna 1: 25-34, 2010.

This work is licensed under a Creative Commons Attribution-NonCommercial-NoDerivatives 4.0 International (CC BY-NC-ND 4.0) License. 